\title{
Cerebrovascular Hemodynamics during and after Recovery from Acute Asphyxia in the Newborn Dog
}

\author{
ANDREW J. MCPHEE, UMA R. KOTAGAL, AND LEONARD I. KLEINMAN
}

Department of Pediatrics, Newborn Division, University of Cincinnati, Cincinnati, Ohio 45267-0541

\begin{abstract}
Cerebrovascular volume and transmural pressure loads accompanying acute increases in cerebral blood flow are implicated in the pathogenesis of periventricular-intraventricular hemorrhage in preterm infants. An acute increase in cerebral blood flow would be expected during acute recovery from asphyxia. Therefore, cerebrovascular hemodynamics, including flow (microspheres), were studied during and after acute recovery from asphyxia in seven newborn dogs in order to study the determinants of these volume and pressure loads. During the acute recovery phase, cerebral hemispheric blood flow was $\mathbf{6 9 . 6}$ $\pm 10 \mathrm{ml} / 100 \mathrm{~g} / \mathrm{min}$ (mean $\pm \mathrm{SEM}$ ) representing a $250 \%$ increase from baseline values of $19.9 \pm 1.8 \mathrm{ml} / 100 \mathrm{~g} / \mathrm{min}$ $(p<0.005)$, while combined cerebellar-brainstem flow was $204.3 \pm 19.3 \mathrm{ml} / 100 \mathrm{~g} / \mathrm{min}$ representing a $536 \%$ increase from baseline values of $32.0 \pm 1.5 \mathrm{ml} / 100 \mathrm{~g} / \mathrm{min}$ $(p<0.005)$. Blood flow to both areas had returned to baseline levels $20 \mathrm{~min}$ after the onset of recovery. Associated with this cerebral hyperemia was an acute increase in mean arterial pressure from $21.3 \pm 4.5 \mathrm{~mm} \mathrm{Hg}$ at end asphyxia to $69.5 \pm 6.0 \mathrm{~mm} \mathrm{Hg}$ at peak recovery $(p<$ 0.01 , and parallel acute increases in sagittal sinus pressure (from $4.0 \pm 0.4$ to $14.6 \pm 1.9 \mathrm{~mm} \mathrm{Hg}, p<0.01$ ) and cerebrospinal fluid pressure (from $3.8 \pm 0.4$ to $14.3 \pm 1.9$ $\mathrm{mm} \mathrm{Hg}, p<0.01$ ). Central venous pressure fell from 4.3 $\pm 0.6 \mathrm{~mm} \mathrm{Hg}$ at end asphyxia to $1.6 \pm 0.5 \mathrm{~mm} \mathrm{Hg}$, and thus is not a determinant of the elevation in sagittal sinus pressure. All of these pressure changes were attained within 20-30 s of the onset of recovery. Assuming that acute changes in cerebrospinal fluid pressure reflect acute changes in cerebrovascular volume via the cranial compliance (volume/pressure) relationship, then the acute $(<30$ s) elevation of cerebrospinal fluid pressure reflects an acute cerebrovascular volume load. Also, the elevated cerebrospinal fluid pressure considerably modifies both the cerebral arterial and venous transmural pressures. The cranial compliance relationship thus plays a key role in determining both volume and transmural pressure loads. We propose that the combination of a high cranial compliance and frequent acute cerebral flow/volume loads may be involved in the pathogenesis of periventricular-intraventricular hemorrhage in premature newborn infants. (Pediatr Res 19: 645-650, 1985)
\end{abstract}

Received October 12, 1984; accepted February 20, 1985.

Reprint requests Dr. Andrew J. McPhee, Department of Pediatrics, Newborn Division, University of Cincinnati, 231 Bethesda Avenue, Cincinnati, OH 45267 0541 .

Supported by a grant from the Southwestern Ohio Branch of the American Heart Association, and by DRR Clinfo Grant RR 68-22 of the General Clinical Reseach Center, University of Cincinnati Medical Center.
Abbreviations

CBF, cerebral blood flow

CSFP, cerebrospinal fluid pressure

SSP, sagittal sinus pressure

MAP, mean arterial pressure

CPP, cerebral perfusion pressure

CVP, central venous pressure

Acute increases in CBF are implicated in the pathogenesis of periventricular-intraventricular hemorrhage in preterm infants $(1,2)$. The theoretical mechanisms by which acute increases in CBF are said to produce periventricular-intraventricular hemorrhage involves the development of excessive volume and transmural pressure loads in the delicate microvasculature of the periventricular germinal matrix. Vessel rupture and hemorrhage occurs if the transmural pressure load exceeds a certain critical pressure. Although the production of microvascular volume and transmural pressure load is central to this theory of the pathogenesis of periventricular-intraventricular hemorrhage, no studies to date have looked at the cerebrovascular hemodynamics and the determinants of these volume and pressure loads during an acute increase in CBF.

The aim of our study was to examine cerebrovascular hemodynamics during an acute increase in CBF in the newborn dog, and recovery from an acute asphyxic insult was chosen for the study because an acute increase in $\mathrm{CBF}$ was expected. The dynamic alterations in CSFP, SSP, CVP, MAP, and CPP prior to and during this acute recovery phase were studied. Conclusions concerning cerebral vascular volume loads and transmural pressure loads were drawn from these acute pressure changes.

\section{MATERIALS AND METHODS}

Animal preparation. Seven newborn mongrel dogs, age 1-6 days, weighing $404 \pm 22 \mathrm{~g}$ (mean \pm SEM) were studied. After being weighed, each dog was anesthetized with intravenous sodium pentobarbital $(10-20 \mathrm{mg} / \mathrm{kg}$, Abbott Labs, N. Chicago, IL), paralyzed with pancuronium bromide $(100-200 \mu \mathrm{g} / \mathrm{kg}$, Organon Inc., W. Orange, NJ), intubated orotracheally, and ventilated with a small animal ventilator (Harvard Apparatus, Southwick, MA) to maintain arterial $\mathrm{pO}_{2}$ greater than 50 torr and $\mathrm{pCO}_{2}$ between 35 and 48 torr. Rectal temperature was monitored continuously and servocontrolled to $37^{\circ} \mathrm{C}$ using an overhead radiant heat source. Under local anesthesia ( $2 \%$ lidocaine hydrochloride, Elkins-Sinn, Cherry Hill, NJ) both femoral arteries and veins were cannulated with PE 50 polyethylene catheters (Intramedic, Clay Adams Co., Parsippany, NJ), and used to measure 
MAP, CVP, and for arterial and venous access. To measure cardiac output and organ blood flows using the reference organ modification of the radiolabeled microsphere technique (3), a similar catheter was passed into the left ventricle via the left carotid artery and used for microsphere injection, while one of the femoral artery catheters was used as the reference organ withdrawal catheter.

Following placement of these catheters, the dog was placed in the left lateral position and the anterior fontanelle was exposed via a small skin incision. The sagittal sinus was then cannulated with a 23-gauge Teflon cannula (Angiocath, Deseret Co., Sandy, UT), and this cannula was used to measure SSP. Finally, a 23gauge needle was passed percutaneously into the cisterna magna and used to measure CSFP. Thereafter a $45-\mathrm{min}$ recovery period preceded the onset of the study. The animal remained in the left lateral position for the duration of the study in order to eliminate positional hydrostatic pressure differences, and all pressure zero calibrations were referenced to the midspinal level with the animal in this position.

Experimental protocol and methods. The experimental protocol involved four sequential study periods (I-IV) as shown in Figure 1. Following baseline measurements (period I), each animal was subjected to an asphyxic insult produced by discontinuing mechanical ventilation. A $71 / 2$-min asphyxic insult was planned. However, severe hypotension and bradycardia forced foreshortening of the length of the insult in several animals. Mechanical ventilation was recommenced at the end of the insult, and period II measurements were made in the ensuing acute recovery phase. Periods III and IV represent later recovery periods 20 and $40 \mathrm{~min}$ after period II, respectively. In two animals, period IV measurements were not performed because microsphere chamber damage resulted in considerable leakage and injection of insufficient microspheres.

SSP, CSFP, CVP, and systemic arterial pressure were measured and recorded continuously using precalibrated pressure transducers (Gould Statham P23 ID, Gould Inc., Cleveland, $\mathrm{OH}$ ) and a four channel amplified recorder (Gould Inc.). MAP was calculated from the arterial tracing as $1 / 3$ systolic plus $2 / 3$ diastolic pressure. CPP was calculated as MAP minus SSP. Because period II is a period of marked changes in these pressures, the dynamic alterations in cerebrovascular hemodynamics during this period were further delineated by determining pressures immediately prior to the onset of recovery and at their individual peaks (or nadir in the case of CVP), during the recovery. These are termed the pre-II and II pressures, respectively. The time course of each of these pressure changes was also determined.

Arterial $\mathrm{pH}, \mathrm{pCO}_{2}$, and $\mathrm{pO}_{2}$ were determined at each study period (Radiometer BMS 3 Mark 2 analyzer, Copenhagen, Denmark), as was the hematocrit. Measurements were made immediately before the flow measurements, except in period II, where the specimen was taken at the end of the flow measurement.

Cardiac output and regional CBF measurements were made at each study period using the reference organ modification of the radiolabeled microsphere technique (3). The period II flow measurements were initiated during the rise in MAP associated with the acute recovery. The preparation and use of the microspheres in this laboratory have been outlined previously (4), and follow the principles and recommendations of Heymann et al (5). Briefly, four species of $15 \mu$ microsphere were used $\left({ }^{85} \mathrm{Sr}\right.$, ${ }^{141} \mathrm{Ce},{ }^{95} \mathrm{Nb}$, and ${ }^{51} \mathrm{Cr}$ ), with $300,000-350,000$ microspheres being injected at each study period. The order of the microsphere injections in a particular experiment was determined from a computer generated random table. The reference organ withdrawal rate was $1.58 \mathrm{ml} / \mathrm{min}$ and the withdrawal was started 10 $\mathrm{s}$ before the microsphere injection and continued for $90 \mathrm{~s}$ after the injection. Well mixed microspheres were injected into the left ventricle over $10-15 \mathrm{~s}$ using $0.5-0.75 \mathrm{ml} \mathrm{N}$ saline to flush the injection chamber. The left ventricular catheter was further flushed with a small volume of $\mathrm{N}$ saline. Previous studies in our laboratory using simultaneous brachial and femoral arterial withdrawals and bilateral femoral arterial withdrawals have documented good microsphere mixing with this technique (4). After each flow determination, $3 \mathrm{ml}$ of adult dog whole blood was infused over several minutes to replace the arterial blood gas and withdrawal sample.

Although the focus of the study was on the cerebrovascular events surrounding the acute recovery period, CBF measurements were not made at the end of asphyxia because of technical problems associated with the use of the microsphere technique under these conditions. These problems include difficulty of obtaining the reference organ withdrawal sample during profound hypotension, and incomplete sampling because of the low cardiac output. In addition, the considerable variability in the individual animal's response to asphyxia presented difficulties in deciding the timing of the onset of the measurement.

At the conclusion of the study the animal was rapidly euthanized with an intravenous bolus of $50 \mathrm{mg}$ sodium pentobarbital, and positions of the left ventricular and central venous catheters were verified. The brain was then removed and divided into right and left cerebral hemispheres, cerebellum, (including midbrain) and brainstem, and these tissues were processed and counted along with the injection chamber and reference organ withdrawal samples. These counts were then used to determine cardiac output and regional CBF measurements for each cerebral hemisphere and for the combined cerebellum and brainstem using procedures previously reported (4). Insufficient microspheres in brainstem samples (less than 400) prevented separation of flows in the infratentorial regions. Finally, absolute and percentage change from baseline (period I) of combined cerebral hemispheric and combined cerebellar-brainstem blood flows were calculated for periods II, III, and IV.

Statistical analysis. One way analysis of variance with repeated measures and Newman-Keuls multiple comparison were used to analyze sequential pressure, flow and biochemical data for periods I to IV. Results of SSP, CSFP, CVP, MAP, and CPP at the end of the asphyxic period (pre-II pressure measurements), and during the acute recovery (II pressure measurements) were incuded in the analysis of pressure variables. The two missing data points for period IV were estimated using standard tests (6) so that the analyses could be performed as a repeated measures analysis. The comparison of combined cerebral hemispheric flows to combined cerebellar-brainstem flow within each period, the comparison of right hemispheric to left hemispheric flows, and the comparison of percentage change from baseline for each region in period II versus I was performed by paired $t$ test analysis. All results are presented as mean \pm SEM.

\section{RESULTS}

The duration of the asphyxic insult varied from 5 to $7 \frac{1}{2} \mathrm{~min}$ (mean $61 / 2 \mathrm{~min}$ ) with shorter asphyxic insults being dictated by the development of severe bradycardia and hypotension.

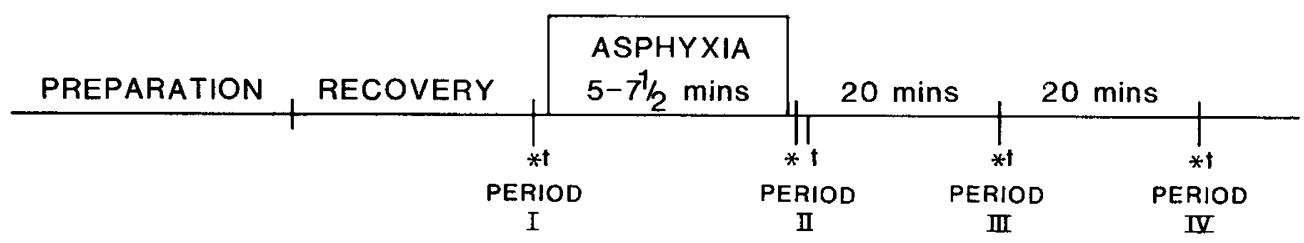

Fig. 1. Experimental protocol. SSP, CSFP, CVP, and MAP were determined at each microsphere injection period $\left({ }^{*}\right)$. Arterial blood gas and hematocrit ( $\dagger$ ) were measured immediately prior to the flow studies in periods I, III and IV, and immediately after the flow study in period II. 
The results of arterial $\mathrm{pH}, \mathrm{pCO}_{2}, \mathrm{pO}_{2}$, and hematocrit are shown in Table 1. Arterial $\mathrm{pO}_{2}$ varied considerably, but was more than 50 torr in all animals. This is above the level of $\mathrm{pO}_{2}$ expected to influence CBF in this model (7). As expected, there was significant acidosis and hypercarbia in period II $(p<0.005$ for $\mathrm{pH}$ and $\mathrm{pCO}_{2}$ ). Hematocrit values did not vary during the study, and arterial $\mathrm{pCO}_{2}$ in periods I, III, and IV did not differ. In addition, $\mathrm{pCO}_{2}$ levels varied by less than 5 torr in each animal during periods I, III, and IV.

Regional CBF results for the combined cerebral hemispheres and the combined cerebellar-brainstem are presented in Figure 2. Blood flow to both regions was markedly elevated during the acute recovery period (period II) compared to all other periods $(p<0.005)$. Cerebral hemispheric blood flow was $69.6 \pm 10 \mathrm{ml} /$ $100 \mathrm{~g} / \mathrm{min}$ in period II, representing a $250 \pm 39 \%$ increase from the baseline (period I) levels of $19.9 \pm 1.8 \mathrm{ml} / 100 \mathrm{~g} / \mathrm{min}$, and had returned to baseline levels in period III $(21.6 \pm 1.7 \mathrm{ml} / 100$ $\mathrm{g} / \mathrm{min})$ and period IV $(20.4 \pm 1.8 \mathrm{ml} / 100 \mathrm{~g} / \mathrm{min})$. Blood flow to the combined cerebellar-brainstem region was $204.2 \pm 19.3 \mathrm{ml} /$ $100 \mathrm{~g} / \mathrm{min}$ in period II, representing a $536 \pm 29 \%$ increase from the baseline (period I) levels of $32.0 \pm 1.5 \mathrm{ml} / 100 \mathrm{~g} / \mathrm{min}$, and had returned to baseline levels in period III $(37.8 \pm 4.3 \mathrm{ml} / 100$ $\mathrm{g} / \mathrm{min})$ and period IV $(32.3 \mathrm{ml} / 100 \mathrm{~g} / \mathrm{min})$. In each study period, blood flow to the combined cerebellar-brainstem region was significantly elevated with respect to flow to the cerebral hemispheres $(p<0.005)$, and the percentage increase from baseline (period I) for the cerebellar-brainstem region in period II is significantly greater than that for the cerebral hemispheres $(p<$ 0.005).

The effect of the left carotid artery ligation on differential hemispheric blood flow was determined by comparing the paired right and left hemispheric blood flows in each period. The results are presented in Table 2 and show that left hemispheric flows were consistently less than right hemispheric flows, except in period IV where only five comparisons were available. However, as also shown in Table 2, when the flows to the individual hemispheres were considered, the results of the comparisons of period II to all other periods were essentially the same as those obtained when the hemispheric flows were combined. In addition, the comparison of infratentorial flows to the flows to either hemisphere produced significance similar to that obtained when the combined flows were considered. Thus, despite an effect of carotid ligation on differential hemispheric blood flow, the responses of the individual hemispheres during the study were similar, and similar results are obtained when either the individual or combined results were used in the flow analyses.

Cardiac output did not differ during the study, being $190 \pm$ $21,189 \pm 49,177 \pm 29$, and $162 \pm 29 \mathrm{ml} / \mathrm{kg} / \mathrm{min}$ for periods II to IV, respectively.

The dynamic changes in cerebrovascular pressures during the acute recovery phase (period II) were of particular interest in this study. These changes are illustrated by the representative tracing shown in Figure 3, and by comparison of the pre-II and II pressure measurements in Table 3. Figure 3 is a representative tracing of the pressure changes accompanying asphyxia and early recovery. It shows the moderate hypotension at the end of a $7 \frac{1 / 2-}{1}$ min asphyxic insult in this animal, and the abrupt increase in systemic arterial pressure during the acute recovery period. Associated with this rise in arterial pressure, but following it by some $10-15 \mathrm{~s}$ in this example, is an acute parallel rise in both

Table 1. Physiologic data for periods I-IV (mean \pm SEM)

\begin{tabular}{lcccc}
\hline Period & I & II & III & IV \\
\hline$(n)$ & $(7)$ & $(7)$ & $(7)$ & $(5)$ \\
$\mathrm{pH}$ & $7.41 \pm 0.02$ & $7.20 \pm 0.06^{*}$ & $7.33 \pm 0.03$ & $7.3 \pm 0.04$ \\
$\mathrm{pCO}_{2}$ (torr) & $40.6 \pm 1.6$ & $52.6 \pm 4.4^{*}$ & $40.1 \pm 1.5$ & $39.1 \pm 1.4$ \\
$\mathrm{pO}_{2}$ (torr) & $118.0 \pm 19.3$ & $133.0 \pm 10.7$ & $94.0 \pm 16.1$ & $97.0 \pm 15.9$ \\
$\mathrm{Hematocrit}^{1}$ & $39.0 \pm 2.0$ & $39.0 \pm 2.0$ & $38.0 \pm 1.0$ & $40.0 \pm 2.0$ \\
\hline
\end{tabular}

${ }^{*} p<0.005$ versus all other periods. ANOVA and Newman-Keuls multiple comparison.

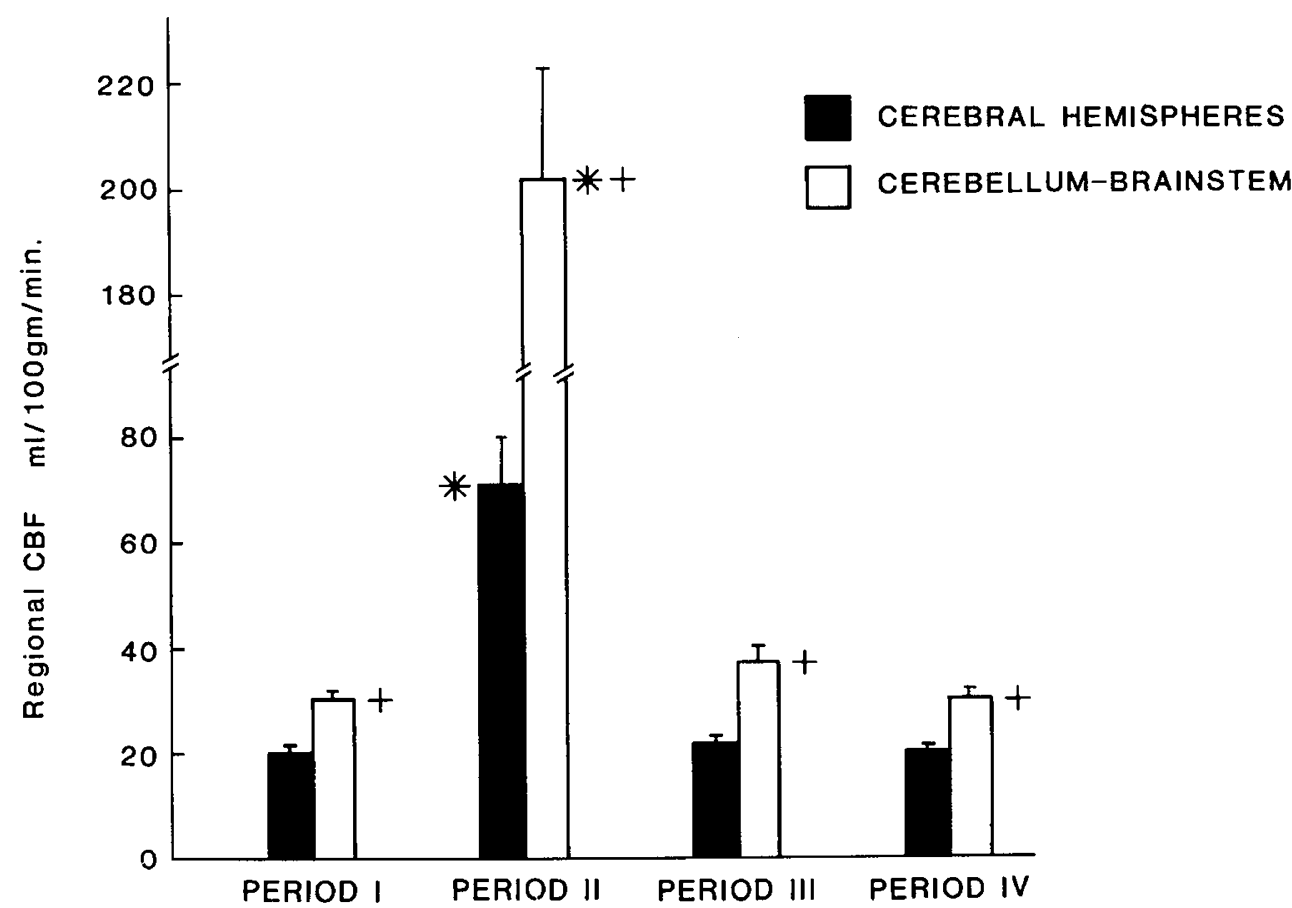

Fig. 2. Cerebral hemispheric blood flow and combined cerebellar-brainstem blood flow for periods I-IV. ${ }^{*} p<0.005$ for cerebral hemispheric and combined cerebellar-brainstem blood flows in period II versus all other periods. (ANOVA with repeated measures and Newman-Keuls multiple comparison). $\dagger p<0.005$ for cerebral hemispheric versus combined cerebellar-brainstem blood flows in each period (paired $t$ test). Mean \pm SEM 
Table 2. Right (R) and left (L) hemispheric blood flows for periods I-IV (mean \pm SEM)

\begin{tabular}{lcccc}
\hline \multicolumn{1}{c}{ Period } & I & II & III & IV \\
\hline $\begin{array}{l}(n) \\
\text { R hemispheric flow } \\
(\mathrm{ml} / 10 \mathrm{~g} / \mathrm{min})\end{array}$ & $(7)$ & $(7)$ & $(7)$ & $(5)$ \\
$\begin{array}{l}\text { L hemispheric flow } \\
(\mathrm{ml} / 100 \mathrm{~g} / \mathrm{min})\end{array}$ & $21.9 \pm 1.9$ & $76.4 \pm 11.7^{*}$ & $23.3 \pm 1.9$ & $22.6 \pm 2.2$ \\
\end{tabular}

${ }^{*} p<0.005$ for period II versus all other periods (ANOVA with repeated measures and Newman-Keuls multiple comparison). $\dagger p<0.01$ versus $\mathbf{R}$ hemispheric flow (paired $t$ test).

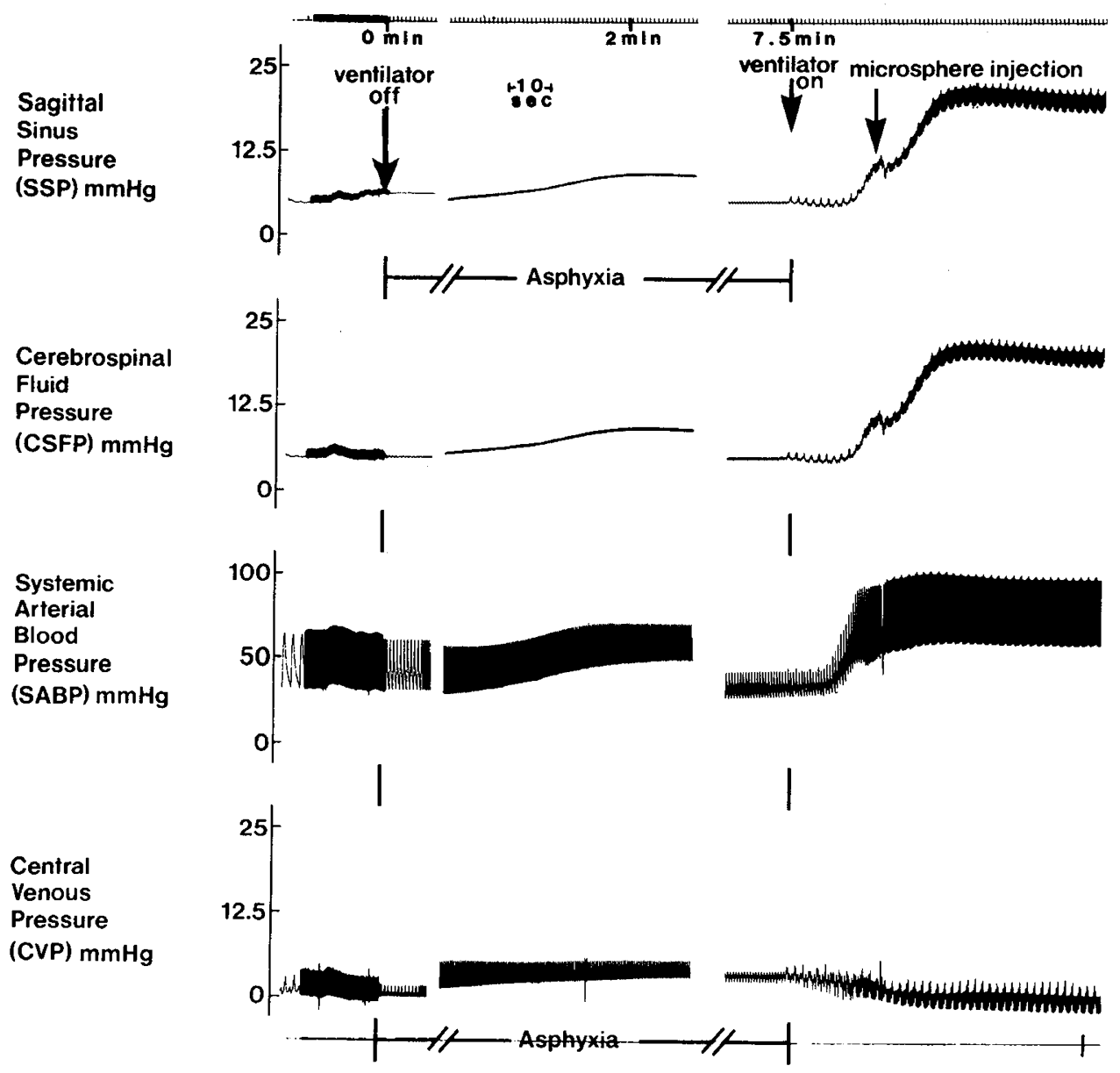

Fig. 3. Representative tracing of SSP, CSFP, systemic arterial blood pressure, and CVP during asphyxia and acute recovery. The tracing is noncontinuous and consists of a panel at the onset of asphyxia ("ventilator off;" $0 \mathrm{~min})$, a panel during asphyxia ( 2 min), and a panel during late asphyxia and acute recovery. The asphyxic insult in this animal lasted $7.5 \mathrm{~min}$, and ends with "ventilator on." During the acute recovery, there is an abrupt increase in systemic ABP, equal increases in SSP and CSFP, and a decrease in CVP. The pre-II and II pressure measurements (see text and table 2) are taken just prior to the end of the asphyxia (pre-II pressures), and at the peak or nadir of the individual pressure changes during recovery (II pressures).

CSFP and SSP. CVP, elevated with respect to baseline at the end of the asphyxic insult, falls during the acute recovery period, and thus is not a determinant of the rise in SSP.

Table 3 shows the results of the MAP, SSP, CSFP, CPP, and CVP measurements for periods I to IV for all animals, and includes the pressure measurements at the end of the asphyxia (pre-II measurements) and during the acute recovery (II measurements). There were no significant differences between individual pressure measurements in periods I, III, and IV. The baseline (period I) MAP of $45.7 \pm 4.5 \mathrm{~mm} \mathrm{Hg}$ is in agreement with previous experience in barbiturate anesthetized dogs of this age (8). By the end of the asphyxia (pre-II), MAP had fallen to $21.3 \pm 4.5 \mathrm{~mm} \mathrm{Hg}(p<0.01$ versus all other periods), but increased abruptly during recovery to peak at $69.6 \pm 6.0 \mathrm{~mm} \mathrm{Hg}$ ( $p<0.01$ versus all other periods). Associated with this increase in MAP, SSP increased from $4.0 \pm 0.4 \mathrm{~mm} \mathrm{Hg}$ (pre-II) to 14.6 $\pm 1.9 \mathrm{~mm} \mathrm{Hg}$ (II) and CSFP increased from $3.8 \pm 0.4 \mathrm{~mm} \mathrm{Hg}$ (pre-II) to $14.3 \pm 1.9 \mathrm{~mm} \mathrm{Hg}$ (II), with both increases being significant $(p<0.01)$. The close relationship between SSP and CSFP, as illustrated in Figure 3, was such that the difference between these pressures remained small (less than $1 \mathrm{~mm} \mathrm{Hg}$ ) at all times. Finally CVP fell from an end asphyxia level of $4.3 \pm$ $0.6 \mathrm{~mm} \mathrm{Hg}$ (pre-II), to $1.6 \pm 0.5 \mathrm{~mm} \mathrm{Hg}$ during the acute recovery $(p<0.01)$. These changes in MAP, SSP, CSFP, and CVP were all attained within $20-30 \mathrm{~s}$ of the onset of recovery. The CPP during the acute recovery (represented by the II results) was calculated as the peak MAP minus the peak SSP. Note however, that the time lag between the MAP peak and the SSP peak, as illustrated in Figure 3, results in a briefly higher CPP during the early phase of the acute recovery. A similar time lag between these pressure peaks was seen in four of the seven animals (maximum lag 10-15 s). 
Table 3. Pressure data for periods I-IV (mean \pm SEM)

\begin{tabular}{|c|c|c|c|c|c|}
\hline Period & I & Pre-II* & $\mathrm{II}^{*}$ & III & IV \\
\hline$(n)$ & (7) & (7) & (7) & (7) & (5) \\
\hline $\mathrm{MAP}(\mathrm{mm} \mathrm{Hg})$ & $45.7 \pm 4.3$ & $21.3 \pm 4.5 \dagger$ & $69.6 \pm 4.0 \dagger$ & $44.6 \pm 5.0$ & $45.3 \pm 7.3$ \\
\hline $\mathrm{SSP}(\mathrm{mm} \mathrm{Hg})$ & $4.3 \pm 0.6$ & $4.0 \pm 0.4$ & $14.6 \pm 1.9 \dagger$ & $2.9 \pm 0.3$ & $2.7 \pm 0.3$ \\
\hline $\mathrm{CSFP}(\mathrm{mm} \mathrm{Hg})$ & $4.1 \pm 0.6$ & $3.8 \pm 0.4$ & $14.3 \pm 1.9 \dagger$ & $2.8 \pm 0.3$ & $2.5 \pm 0.3$ \\
\hline $\mathrm{CPP}(\mathrm{mm} \mathrm{Hg})$ & $41.4 \pm 4.4$ & $17.3 \pm 4.4 \dagger$ & $55.0 \pm 5.4 \dagger$ & $41.8 \pm 5.1$ & $42.7 \pm 7.4$ \\
\hline $\mathrm{CVP}(\mathrm{mm} \mathrm{Hg})$ & $1.0 \pm 0.3$ & $4.3 \pm 0.6 \dagger$ & $1.6 \pm 0.5$ & $0.6 \pm 0.4$ & $0.4 \pm 0.4$ \\
\hline
\end{tabular}

* See text for derivation of these values.

$\dagger p<0.01$ versus all other periods. ANOVA with repeated measures and Newman-Keuls multiple comparison.

\section{DISCUSSION}

Changes in cerebrovascular hemodynamics accompanying acute increases in CBF are implicated in the pathogenesis of periventricular-intraventricular hemorrhage. The correlation of hemodynamic changes accompanying an acute increase in $\mathrm{CBF}$ is hampered by current technical difficulties involved in the continuous measurement of $\mathrm{CBF}$, and thus changes in flow can be determined only by comparison of serial measurements. As mentioned earlier, technical difficulties precluded CBF measurements at the end of asphyxia in our study. However, previous work by Hernandez et al. $(9,10)$ and Hellman et al. (11) have studied CBF during asphyxia using a similar asphyxia model as ours, namely, respiratory cessation in paralyzed ventilated newborn dogs. These studies allow conclusions concerning CBF at the end of the asphyxic period in our study, and when combined with our results during the acute recovery phase, provide an estimate of the magnitude of the increase in $\mathrm{CBF}$ accompanying the recovery. Thus, Hernandez et al. $(9,10)$ measured regional $\mathrm{CBF}$ at 5 min of asphyxia using ${ }^{14} \mathrm{C}$-iodoantipyrine autoradiography and showed that blood flow was less than baseline for all areas except the pons, medulla, and spinal cord where it was 30 , 80 , and $83 \%$ above baseline, respectively. Recently, Hellman et al. (11) extended the asphyxic insult to 7-8 min duration and using the same autoradiographic technique showed that regional CBF was less than or equal to baseline for all areas studied. On the basis of these studies, it seems reasonable to assume that regional CBF at the end of the asphyxic period in our study was less than or equal to baseline.

The baseline regional CBF results and the increased flow to infratentorial regions in our study concurs with previous data in newborn dogs $(9,12-14)$. During the acute recovery phase the cerebral hemispheric and the combined cerebellar-brainstem flows increased $250 \pm 39$ and $536 \pm 29 \%$ above baseline, respectively, with the percentage increase to the brainstem-cerebellum being significantly greater than that to the hemispheres $(p<0.005)$. These flow measurements were completed within 2 min of the onset of recovery and thus, if the above mentioned assumption concerning flow at the end of the asphyxic period is valid, then these high flows developed rapidly. Also, because the measurement of $\mathrm{CBF}$ under such nonsteady state conditions as period II is confined to giving average or integrated flows over the study period, instantaneous flows may be higher. At 20 and $40 \mathrm{~min}$ after the onset of the acute recovery, blood flow had returned to baseline levels, indicating a short $(<20 \mathrm{~min})$ postasphyxic hyperemia.

The relative contribution of each of the various known determinants of CBF to the increased flows in period $\mathrm{II}$, and to the greater proportional increase in flow to the cerebellar-brainstem region, is speculative because none of the determinants is independently controlled, and because most are actually changing during the study period. However, it is known that an acute increase in arterial pressure results in a transient increase in CBF (15). The magnitude of the increase in CBF accompanying an acute increase in arterial pressure may depend on the tone of the cerebral resistance vessels at the time of the pressure rise, with high flows occurring when the vascular resistance at the time of the pressure rise is low. Hypercarbia (13), hypoxia $(7,16)$, and hypotension (17) are each known to lower cerebral vascular resistance under steady state conditions in the newborn dog. In our study, asphyxia is associated with significant hypotension, while studies by others in newborn dogs have shown marked hypercarbia and hypoxia during asphyxic insults of similar duration $(9,10)$. Therefore the high flows seen in period II may be due to the marked acute increase in arterial pressure at a time when the vascular resistance is low due to the combined effects of the immediately preceding hypoxia, hypercarbia, and hypotension. The greater proportional increase in flow to infratentorial regions presumably reflects greater sensitivity of the resistance vessels in these areas to one or more of these flow determinants. Such regional differences in sensitivity are known to exist under steady state conditions of hypoxia and hypercarbia (16). Overall, the flow portion of our study confirmed our expectation that acute recovery from asphyxia would be associated with a marked acute increase in $\mathrm{CBF}$, although the specific determinants of this increase in CBF are speculative.

The behavior of the cerebrovascular circuit during the acute postasphyxial increase in blood flow was the main focus of this study. A cerebral microvascular volume load with the subsequent development of critical transmural pressures is central to any mechanical stress theory of microvascular rupture. Microvascular volumes and transmural pressures are obviously difficult to measure. However, the changes in MAP, SSP, and CSFP during the acute increase in CBF accompanying acute recovery from asphyxia allow certain conclusions concerning volume and transmural pressure loads in the cerebrovascular circuit during this acute hyperemia.

At any moment in time, the intracranial volume is the sum of brain tissue volume, cerebral blood volume, and CSF volume, with the total intracranial volume being related to intracranial pressure (i.e. CSFP) via the cranial compliance relationship (18). Changes in CSFP therefore reflect changes in intracranial volume with the magnitude of the pressure change accompanying a given volume change being a function of the cranial compliance relationship. Increases in cerebral blood volume are known to accompany hypercarbic- and hypoxic-induced increases in $\mathrm{CBF}$ (19-21), and to accompany alterations in venous pressure in the open skull preparation (22). In the latter, visible swelling of the brain occurred with obstruction of venous drainage, but brain water content did not increase, implying an increase in cerebral blood volume as the cause of the brain swelling. Assuming that neither the brain tissue volume nor the CSF volume are likely to increase during the brief time frame of the acute recovery period in our study, then the marked elevation in CSFP during our study indicates an increase in cerebral vascular volume. The compartment of the cerebral circulation in which this volume load develops is not addressed by this study.

Cerebral vascular transmural pressures accompanying the high flow/volume load can be determined from the MAP, SSP, and CSFP changes. The cerebral venous transmural pressure is the SSP minus CSFP, while the cerebral arterial transmural pressure is represented by MAP minus CSFP. In our experiment, SSP and CSFP are closely related, and parallel each other at all times, such that the calculated venous transmural pressure is always small. This close relationship between cerebral venous pressure and CSFP is known to exist over a considerable range of pressures 
$(23,24)$, and is a result of the nature of cerebral venous hemodynamics, best described as a Starling resistor or vascular waterfall $(23,25)$. Briefly stated, cerebral venous patency (and flow) requires cerebral venous pressure to be slightly greater than CSFP; a primary elevation of CSFP results in a tendency to cerebral venous compression with decreased outflow eventually leading to an increase in venous pressure and reestablishment of flow when the cerebral venous pressure exceeds CSFP; conversely, a primary increase in cerebral venous pressure increases the volume of the venous compartment of the total cranial volume, and hence the intracranial pressure (CSFP). The cranial compliance relationship assumes a central role in this cerebral venous pressure/CSFP interaction, because the compliance relationship dictates pressure changes occurring with volume changes, and vice versa, for either the cerebral venous or cerebrospinal fluid compartment.

Because of the close relationship between SSP and CSFP it follows that the cerebral perfusion pressure (i.e. MAP minus SSP) must always approximate the cerebral arterial transmural pressure (i.e. MAP minus CSFP). In our study, cerebral perfusion pressure (and therefore arterial transmural pressure) increased from $17.3 \pm 4.4$ to $55.0 \pm 5.4 \mathrm{~mm} \mathrm{Hg}$ during the first $20-30 \mathrm{~s}$ of the acute recovery period. Because of the time lag between the MAP and the SSP/CSFP peaks seen in four of the seven animals, the perfusion pressure (and the transmural pressure) exceeded this value for a brief period of time in these animals, and was attained earlier. The degree to which this acute increase in arterial transmural pressures is transmitted to the arterial side of the microvasculature, will depend on the cerebral vascular resistance, as determined by the tone of the resistance arterioles. For the reasons mentioned earlier, the tone of these resistance vessels is probably very low, and hence large transmural pressures may develop on the arterial side of the microcirculation during this acute high flow period. These elevated microvascular transmural pressures may be sufficient to cause vessel rupture and hemorrhage in the germinal matrix. Note, however, that the elevation of CSFP accompanying the acute increase in CBF considerably modifies the magnitude of the arterial transmural pressure load, and as such provides some protection against the development of large transmural pressure loads. The role of the cranial compliance relationship in the development of this increase in CSFP is important; indeed, if cranial compliance was near zero (i.e. rigid, fixed skull), then even small changes in cerebrovascular volume accompanying an acute increase in flow would be associated with large changes in intracranial pressure, thus markedly limiting changes in arterial transmural pressures. A more compliant cranium, such as that seen in the premature infant would afford less protection.

Finally, this study only considers the hemodynamic changes in the cerebrovascular circuit as a whole. If the conclusions concerning the development of large transmural pressures on the arterial side of the microvasculature are valid, and if these pressures do result in vessel rupture and hemorrhage in the germinal matrix, then additional local factors such as intrinsic microvascular weakness and/or differential (exaggerated) transmission of arterial transmural pressures must be invoked to explain the selective occurrence of hemorrhage in the germinal matrix.

In summary, acute recovery from a brief asphyxic insult is associated with a marked acute increase in regional CBF, systemic hypertension, and an intense elevation of both CVP and CSFP. The implications of the dynamic changes in MAP, CSFP, and SSP during this high flow state are discussed, and provide insight into the significance of the cranial compliance relationship as it relates to the development of cerebral vascular volume and transmural pressure loads. Assuming some intrinsic vulnerability of the microvasculature of the germinal matrix, we propose that the combination of acute increases in CBF and a relatively high cranial compliance may be important in the pathogenesis of periventricular-intraventricular hemorrhage in premature newborn infants.

Acknowledgments. The authors thank Ms. Katherine Denham for her technical assistance and Ms. Alesia Mattar for secretarial assistance in the preparation of the manuscript.

\section{REFERENCES}

1. Hambleton G, Wigglesworth JS 1976 Origin of intraventricular hemorrhage in the preterm infant. Arch Dis Child 51:651-659

2. Wigglesworth JS, Pape KE 1978 An integrated model for haemorrhagic and ischaemic lesions in the newborn brain. Early Hum Dev 2:179-191

3. Archie JP Jr, Fixler DE, Ullyot DJ, Hoffman JI, Utley JR, Carison EL 1973 Measurement of cardiac output with and organ trapping of radioactive microspheres. J Appl Physiol 35:148-154

4. Camp D, Kotagal UR, Kleinman LI 1982 Preservation of cerebral autoregulation in the unanesthetized hypoxemic newborn dog. Brain Res 241:207213

5. Heymann MA, Payne BD, Hoffman JIE, Rudolph AM 1977 Blood flow measurements with radionuclide-labeled particles. Prog Cardiovasc Dis 20:55-79

6. Snedecor GW, Cochran WG 1967 Two-way classifications. In: Statistical Methods, 6th ed. Iowa State University Press, Ames, IA, pp 299-338

7. Vannucci RC, Hernandez MJ 1980 Perinatal cerebral blood flow. In: Mead Johnson Symposium on Perinatal and Developmental Medicine, no 17, pp $17-29$

8. Kleinman LI, Lubbe RJ 1972 Factors affecting the maturation of glomerular filtration rate and renal plasma flow in the newborn dog. J Physiol 223:395409

9. Hernandez MJ, Hawkins RA, Brennan RW 1980 Regional cerebral blood flow during neonatal asphyxia. In: Passoneau JV, Hawkins RA, Lust WD, Welsh FA (eds) Cerebral Metabolism and Neural Function. Williams \& Wilkins, Baltimore, pp 196-201

10. Hernandez MJ, Hawkins RA, Brennan RW 1982 Sympathetic control of regional cerebral blood flow in the asphyxiate newborn dog. In: Heistad D, Marcus M (eds) Cerebral Blood Flow: Effects of Nerves and Neurotransmitters. Elsevier, North Holland, pp 359-366

11. Hellman J, Batton DG, Nardis EE 1983 Regional cerebral blood flow (rCBF) during asphyxia and recovery in newborn dogs. Pediatr Res 17:364 (abstr)

12. Goddard-Finegold J, Michael LH 1984 Cerebral blood flow and experimental intraventricular hemorrhage. Pediatr Res 18:7-11

13. Hernandez MJ, Brennan RW, Vannucci RC, Bowman GS 1978 Cerebral blood flow and oxygen consumption in the newborn dog. Am J Physiol 234:R209-R215

14. Kennedy C, Grave CD, Jehle JW, Sokoloff L 1972 Changes in blood flow in the component structures of the dog brain during postnatal maturation. J Neurochem 19:2423-2433

15. Busija DW, Heistad DD, Marcus MI 1981 Continuous measurement of cerebral blood flow in anesthetized cats and dogs. Am J Physiol 241:H228234

16. Cavazzuti M, Duffy TE 1982 Regulation of local cerebral blood flow in normal and hypoxic newborn dogs. Ann Neurol 11:247-257

17. Hernandez MJ, Brennan RW, Bowman GS 1980 Autoregulation of cerebral blood flow in the newborn dog. Brain Res 184:199-202

18. Shapiro HM 1975 Intracranial Hypertension: therapeutic and anesthetic considerations. Anesthesiology 43:445-471

19. Jobsis FF, Keizer JH, LaManna JC, Rosenthal M 1977 Reflectance spectrophotometry of cytochrome $a_{3}$ in vivo. J Appl Physiol 43:858-872

20. Risberg J, Ancri D, Ingvar DH 1969 Correlation between cerebral blood volume and cerebral blood flow in the cat. Exp Brain Res 8:321-326

21. Grubb RL, Raichle ME, Eichling JO, Ter-Pogossian MM 1974 The effects of changes in $\mathrm{PaCO}_{2}$ on cerebral blood volume, blood flow, and vascular mean transit time. Stroke 5:630-639

22. Cuypers J, Matakas F, Potolicchio SJ 1976 Effect of central venous pressure on brain tissue pressure and brain volume. J Neurosurg 45:89-94

23. Luce JM, Huseby JS, Kirk W, Butler J 1982 A Starling resistor regulates cerebral venous outflow in dogs. J Appl Physiol 53:1496-1503

24. Rushmer RF, Beckman EL, Lee D 1947 Protection of the cerebral circulation by the cerebrospinal fluid under the influence of radial acceleration. Am J Physiol 151:355-365

25. Permutt S, Riley RL 1963 Hemodynamics of collapsible vessels with tone: the vascular waterfall. J Appl Physiol 18:924-932 